\title{
Train like an athlete: applying exercise interventions to manage type 2 diabetes
}

\author{
Mladen Savikj ${ }^{1}$ (D) Juleen R. Zierath ${ }^{1,2,3}$ (D) \\ Received: 16 January 2020 / Accepted: 12 March 2020 / Published online: 11 June 2020 \\ (C) The Author(s) 2020
}

\begin{abstract}
Exercise elicits high energy demands, stimulating cardiorespiratory function and substrate mobilisation and oxidation. Repeated bouts of exercise lead to whole-body adaptations, which improve athletic performance. Distinct exercise modalities and intensities and nutritional conditions pose specific physiological challenges, subsequently inducing different adaptations to training. Athletes often modify these variables to achieve individualised training goals and maximise performance. Exercise training improves glycaemic control in individuals with type 2 diabetes; however, the precise training regimen that confers the most beneficial metabolic adaptations in this population is unknown. In this review, we discuss how modifying exercise type, intensity and modality and nutritional status affects the beneficial effects of exercise on glycaemic control in individuals with type 2 diabetes. Evidence indicates that greater improvements in glycaemic control can be achieved through combined aerobic and resistance training regimens compared with either training type alone. However, the increased frequency of training and a greater number of exercise bouts during combined programmes could be responsible for apparent advantages over a single training modality. The beneficial effects of aerobic exercise on glycaemic control seem to rise with training intensity, with superior adaptations achieved by high-intensity interval training (HIT). In addition, training with low carbohydrate availability ('training low') improves cardiorespiratory function and skeletal muscle oxidative capacity more than conventional training in healthy untrained individuals. Examinations of various training regimens are warranted to assess the safety, efficacy, feasibility and beneficial effects in the type 2 diabetes population. Just like competitive athletes, individuals with type 2 diabetes should be encouraged to adopt training regimens that improve fitness and metabolism.
\end{abstract}

Keywords Aerobic training $\cdot$ Blood glucose $\cdot$ Exercise $\cdot$ Resistance training $\cdot$ Review $\cdot$ Training intensity $\cdot$ Type 2 diabetes

$\begin{array}{ll}\text { Abbreviations } \\ \text { AMPK } & \text { AMP-activated protein kinase } \\ \mathrm{HIT} & \text { High-intensity interval training } \\ \dot{V} \mathrm{O}_{2 \max } & \begin{array}{l}\text { Maximal oxygen consumption } \\ \end{array} \\ & \text { rate }\end{array}$

Electronic supplementary material The online version of this article (https://doi.org/10.1007/s00125-020-05166-9) contains a slideset of the figures for download, which is available to authorised users.

Juleen R. Zierath

Juleen.Zierath@ki.se

1 Department of Molecular Medicine and Surgery, Integrative Physiology, Karolinska Institutet, 17177 Stockholm, Sweden

2 Department of Physiology and Pharmacology, Integrative Physiology, Karolinska Institutet, Stockholm, Sweden

3 Novo Nordisk Foundation Center for Basic Metabolic Research, Faculty of Health and Medical Sciences, University of Copenhagen, Copenhagen, Denmark
$\dot{V} \mathrm{O}_{2 \text { peak }} \quad$ Peak oxygen consumption rate

Exercise elicits a state of high energy demand, stimulating numerous bodily functions, which then work in concert to maintain energetic homeostasis. Cardiorespiratory function and substrate mobilisation and oxidation rise to meet this challenge (Fig. 1). Respiration accelerates and deepens (frequency $>40$ breaths $/ \mathrm{min}$ and tidal volume $\sim 3-4$ ), increasing effective lung ventilation 20 -fold ( 200 1/min) during intense exercise [1]. Concurrently, cardiac output surges $(20-40 \mathrm{l} / \mathrm{min})$ due to accelerated heart rate and stroke volume (frequency up to 200 beats/min and 


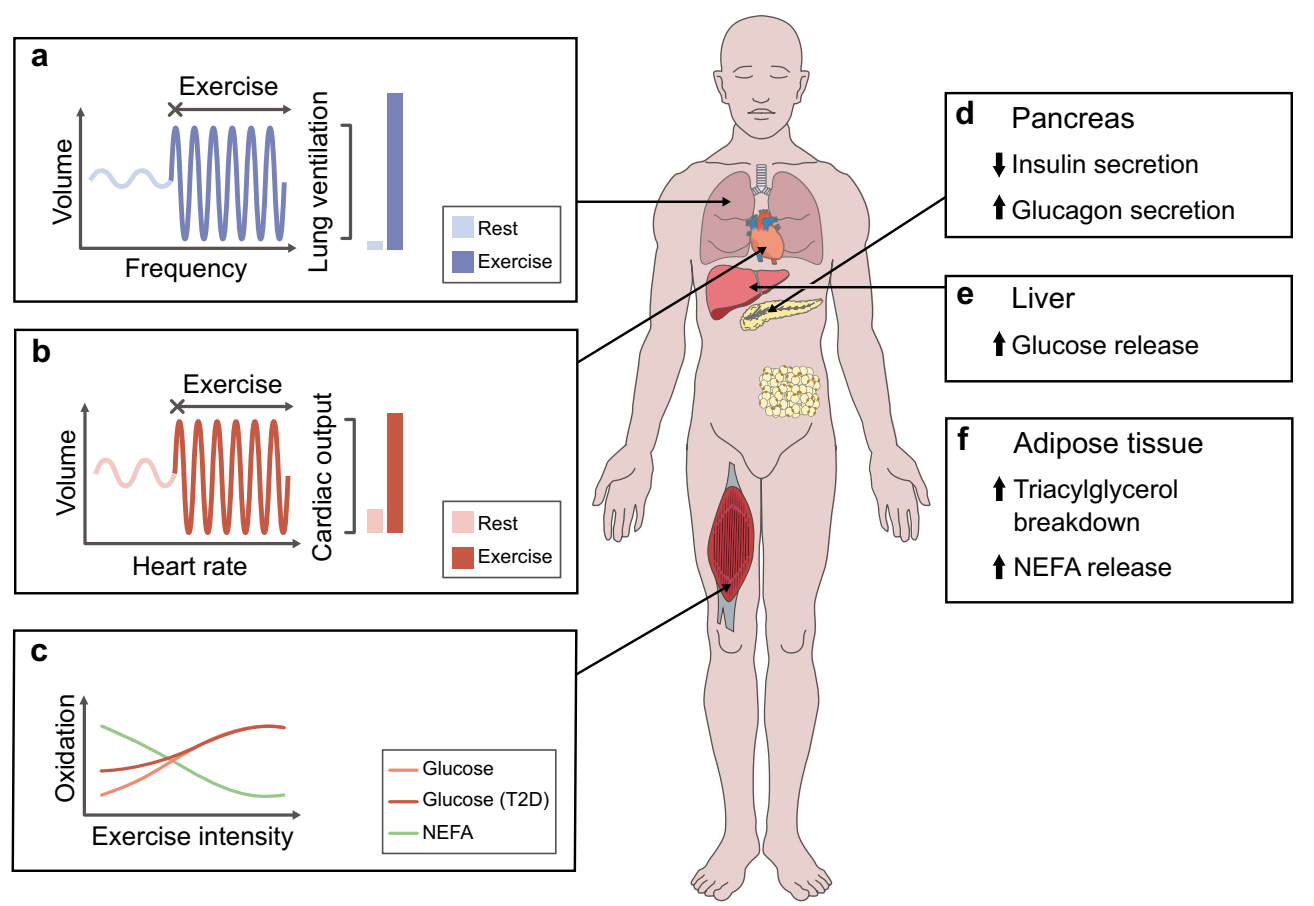

Fig. 1 Acute response to an exercise bout. (a) Once exercise commences, respiratory frequency and tidal volume rise, increasing lung ventilation 20 -fold compared with rest. (b) Similarly, increased heart rate and stroke volume lead to a fivefold higher cardiac output. Increased cardiorespiratory function allows for greater substrate and oxygen delivery to the active skeletal muscle. (c) NEFA are preferentially oxidised by skeletal muscle during low-intensity exercise, whereas glucose is the preferred fuel source during high-intensity exercise. Individuals with type 2 diabetes with hyperglycaemia show a greater reliance on glucose oxidation during lower-intensity exercise compared with healthy control individuals. (d-f) High substrate availability is maintained through glucose and NEFA release by the liver and adipose tissue, respectively, supported by increased glucagon and decreased insulin secretion by the pancreas. T2D, type 2 diabetes. This figure is available as part of a downloadable slideset
60-100\% increase in volume) [1]. Oxygen- and substraterich blood is diverted to active musculature by changes in vascular conductance and the action of the skeletal muscle blood pump [1]. Such stimulation of cardiorespiratory function effectively raises oxygen and substrate delivery to skeletal muscle, providing the means for higher fuel oxidation. The adaptations that occur in response to each exercise bout are quite remarkable, and clinical physiologists have used this knowledge to apply training regimens to improve human health.

Substrate utilisation preference depends on exercise modality, intensity and duration and the nutritional status of the individual. Skeletal muscle relies on glycogen stores and exogenous substrates (blood glucose and nonesterified fatty acids; NEFA) to meet the energetic needs. The relative contribution of glycogen is greater during the initial stages of exercise, while reliance on exogenous substrates rises as the work bout persists [2]. Skeletal muscle glucose uptake rises $\sim 7$-fold during low-intensity and $\sim 20$-fold during higher-intensity exercise [3]. Concurrently, the contribution of blood glucose to substrate oxidation increases from $\sim 10 \%$ initially, to $\sim 30 \%$ during low-intensity and $\sim 40 \%$ during highintensity exercise [3]. NEFA can account for up to $50 \%$ of substrate oxidation during low-intensity exercise [2]. Hence, glucose is the preferred fuel source during highintensity exercise, while NEFA are predominantly utilised during low-intensity exercise. The dependency on glucose oxidation during low-intensity exercise is higher in individuals with type 2 diabetes presenting with hyperglycaemia, with a lower reliance on glycogen [4]. Increased glucose availability is likely to play a role in this shift, as mild glycogen sparing occurs in healthy individuals when exercising during a constant glucose infusion [5]. Hence, this shift towards glucose preference is likely to be relevant only in hyperglycaemic individuals. The relative contribution of exogenous substrates is further modulated by nutritional status. Consuming a highcarbohydrate meal before exercise promotes greater glucose utilisation than a low-carbohydrate meal $[6,7]$. Conversely, NEFA are the predominant fuel source when exercising under fasted conditions [7]. Exercise suppresses insulin release and promotes glucagon secretion, maintaining elevated blood glucose through hepatic glucose release, while adrenergic stimulation maintains elevated NEFA levels by stimulating adipose tissue triacylglycerol breakdown [1]. Hence, external substrate utilisation preference depends on the exercise modality and the timing of 
the training bout and is supported by substrate mobilisation in the adipose tissue and liver.

\section{Expanding performance limits through training}

During training, repeated homeostatic challenges induce cardiorespiratory and skeletal muscle adaptations, which improve athletic performance. Distinct training types (aerobic or resistance) pose specific physiological challenges and induce different adaptations (Fig. 2). Resistance training promotes muscle fibre growth and transformation to a glycolytic phenotype, with increased percentage of fast-twitch fibres and lactate dehydrogenase content, while aerobic training increases mitochondrial density, oxidative enzymes and the relative proportion of slow-twitch fibres [2]. Partly as a result of focused resistance training, elite shot-putters have skeletal muscle composed predominantly $(\sim 60 \%)$ of large, fast-twitch fibres suited to explosive, glycolysis-driven
Fig. 2 Adaptations to different training types. Aerobic training enhances cardiovascular function and promotes skeletal muscle mitochondrial biogenesis, thereby improving exercise endurance.

Resistance training promotes skeletal muscle hypertrophy and increases strength, allowing for more powerful contractions fuelled by glycolysis and supported by higher lactate dehydrogenase content. A combined aerobic and resistance training programme improves endurance and strength/power, albeit to a lesser extent than individual forms of training. However, all training types improve skeletal muscle glucose transport and glycogen synthesis capacity, expanding glycogen stores and improving glycaemic control. This figure is available as part of a downloadable slideset
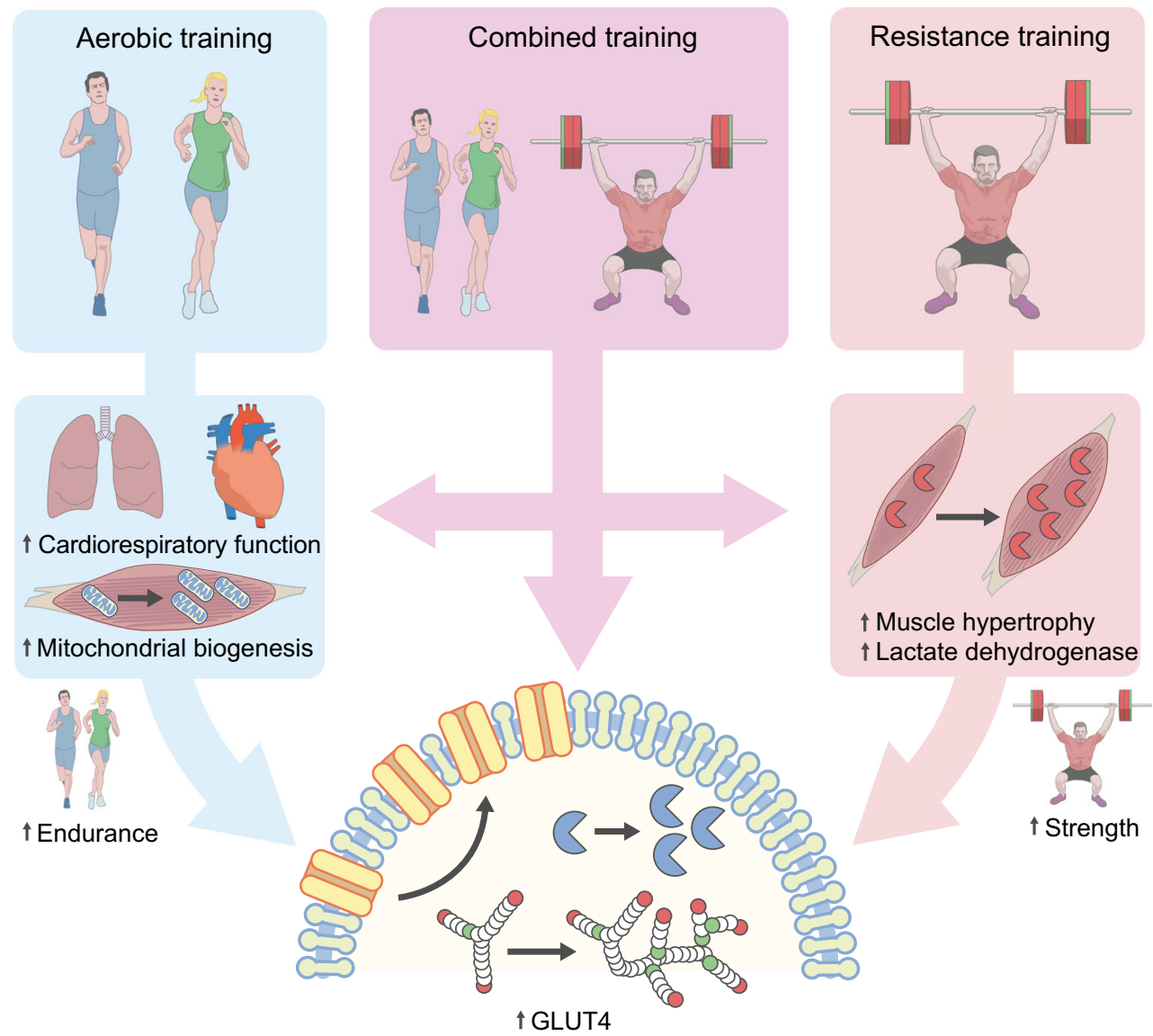

$\uparrow$ Glycogen synthase $\uparrow$ Glycogen stores

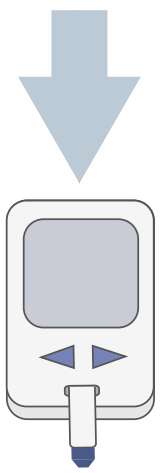

$\uparrow$ Glycaemic control

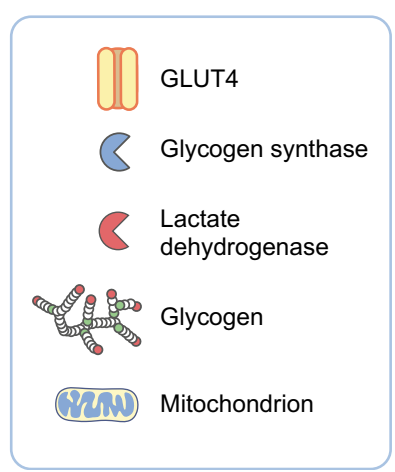


actions, such as propelling $7.25 \mathrm{~kg}$ shot-puts over $20 \mathrm{~m}$ [8]. Conversely, the skeletal muscle of distance runners contains predominantly $(\sim 70 \%)$ slow-twitch fibres and has a high mitochondrial content, allowing prolonged efforts supported by substrate oxidation [8]. Additional cardiorespiratory adaptations allow elite athletes to reach remarkable maximal oxygen consumption rates $\left(\dot{V} \mathrm{O}_{2 \max } ; \sim 80 \mathrm{ml} \mathrm{kg}^{-1} \mathrm{~min}^{-1}\right.$ ) compared with untrained individuals $\left(\sim 40 \mathrm{ml} \mathrm{kg} \mathrm{min}^{-1}\right)$ and endure exceptional workloads [1]. Combined training improves strength and endurance to a lesser extent than focused regimens of either resistance or endurance, respectively [9]. While elite athletic performance is truly remarkable, training adaptations in the general population are more discreet, with fibretype composition and $\dot{V} \mathrm{O}_{2 \max }$ showing modest improvements $[10,11]$. Variations in such improvements are likely to be caused by genetic differences, as training-induced improvements in $\dot{V} \mathrm{O}_{2 \max }$ show notable familial distribution [10]. However, even modest improvements in strength and conditioning are clinically meaningful, as low fitness levels are a relevant predictor of mortality [12]. While an athlete's choice of training depends on performance goals, the primary measure of success of a training regimen for the management of type 2 diabetes is the extent of improvement in glycaemic control, which will be the focus of this review.

\section{Metabolic benefits of different training types}

Exercise increases skeletal muscle glucose uptake by an insulin-independent mechanism [13]. Unsurprisingly, canonical acute responses to exercise, such as increased AMPactivated protein kinase activity and expression of the gene encoding peroxisome proliferator-activated receptor $\gamma$ coactivator $1 \alpha(P G C-1 \alpha$, also known as PPARGC1A) are conserved in insulin-resistant skeletal muscle [14]. Exercise training also restores skeletal muscle mitochondrial function in individuals with type 2 diabetes and induces gains in strength and endurance that are comparable to those observed in healthy individuals [15]. Although insulin sensitivity is also improved through exercise training, it remains lower than that in healthy control individuals [16]. The age of the participant or duration of type 2 diabetes may influence the metabolic adaptations to exercise training, with younger individuals showing greater improvements in metabolic control [17]. Nevertheless, exercise type, intensity and volume and training duration can be modulated to achieve glycaemic improvements [18]. While a complete remission of insulin resistance may not always be achieved, better glycaemic control can be attained by finetuning the training modality.

Participation in any type of training regimen improves glycaemic control [19], but the relative advantage conferred by specific exercise types is less clear. Aerobic training has traditionally been prescribed for type 2 diabetes management, supported by evidence from RCTs showing lower (by $0.5-$ $3.1 \%$; $5.5-33.4 \mathrm{mmol} / \mathrm{mol}$ ) $\mathrm{HbA}_{1 \mathrm{c}}$ levels after 2-6 months of moderate- to high-intensity aerobic training as compared with conventional treatment [20-23]. A meta-analysis of nine RCTs reported a small but consistent and relevant decrease in $\mathrm{HbA}_{1 \mathrm{c}}$ levels of $0.67 \%(\sim 7.5 \mathrm{mmol} / \mathrm{mol})$ in response to aerobic training in individuals with type 2 diabetes [19]. Such beneficial effects on glycaemic control are due to two essential characteristics of aerobic exercise, namely to bypass insulin resistance and increase skeletal muscle glucose uptake and to potentiate insulin sensitivity following an exercise bout [24]. Acute aerobic exercise increases whole-body insulin sensitivity, enhancing skeletal muscle glucose uptake, and this effect persists for $>48 \mathrm{~h}$, while exercise training confers further improvements [2]. The response to both high- and low-dose insulin infusion during a hyperinsulinaemic clamp improves after training (45\% with $0.24 \mathrm{pmol} \mathrm{m}^{-2} \mathrm{~min}^{-1}$ and $17 \%$ with $6 \mathrm{pmol} \mathrm{m} \mathrm{min}^{-1}$ insulin infusion), indicating increased insulin sensitivity and responsiveness [25]. Exercise-induced improvements in insulin action are likely to result from skeletal muscle adaptations, such as increased glycogen stores and synthesis rates, supported by increases in GLUT4 protein content and glycogen synthase activity $[1,2]$. However, improvements acquired through aerobic training may be temporary as fasting glucose and insulin values regress to pre-training levels $\sim 72 \mathrm{~h}$ after the last training bout [26]. Thus, while aerobic training improves insulin sensitivity and glycaemic control, maintaining the exercise regimen may be necessary to preserve these benefits.

A single bout of resistance exercise leads to an acute improvement in glycaemic control, and decreased fasting glucose and insulin levels (by $0.2 \mathrm{mmol} / \mathrm{l}$ and $17.4 \mathrm{pmol} / \mathrm{l} \mathrm{vs}$ pre-training, respectively) the subsequent morning [27]. RCTs show that longer resistance training periods (5-6 months) lead to lower $\mathrm{HbA}_{1 \mathrm{c}}$ levels $(0.4-0.8 \%, 4.1-8.7 \mathrm{mmol} / \mathrm{mol})$ than those observed with standard care in the type 2 diabetes population $[23,28]$. These improvements are likely to be due to skeletal muscle adaptations, as 6 weeks of unilateral lowerlimb resistance training increases glucose uptake (18\%), GLUT4 protein content, and glycogen synthase activity in the trained vs untrained skeletal muscle of individuals with type 2 diabetes [29]. Controlled trials of 4-6 weeks of extensive whole-body training show heightened insulin sensitivity and increased glucose disposal (48\%) during a hyperinsulinaemic clamp at least $48 \mathrm{~h}$ after the last resistance exercise bout [30]. Hence, in addition to aerobic regimens, resistance training regimens are efficacious in improving glycaemic control in individuals with type 2 diabetes.

The relative efficacy of an aerobic, resistance or combined training regimen for improving glycaemic control has been assessed in individuals with type 2 diabetes. An RCT of a 6 month training regimen (aerobic, resistance or both 
combined) showed that $\mathrm{HbA}_{1 \mathrm{c}}$ levels are $0.4-0.9 \%$ (4.1$10.6 \mathrm{mmol} / \mathrm{mol}$ ) lower after any training regimen compared with standard care [23]. A parallel 4 month trial of aerobic or resistance training reported similar reductions in $\mathrm{HbA}_{1 \mathrm{c}}$ levels $(\sim 0.4 \% ; \sim 4.4 \mathrm{mmol} / \mathrm{mol})$, with mild advantage of aerobic over resistance training on improvements in insulin sensitivity (30\% vs $15 \%$ improvement) [31]. However, a combination of aerobic and resistance training reduces $\mathrm{HbA}_{1 \mathrm{c}}$ levels to a greater extent $(0.9 \% ; 10.6 \mathrm{mmol} / \mathrm{mol})$ than either regimen alone $(0.4-0.5 \% ; 4.1-5.5 \mathrm{mmol} / \mathrm{mol})$ [23]. Similarly, an RCT showed greater insulin-sensitising effects of 4 months of combined training compared with aerobic training alone, as evidenced by greater glucose disappearance rates during a hyperinsulinaemic clamp ( $77 \%$ and $20 \%$ increase for combined and aerobic training, respectively) [32]. These differences may partly be accounted for by larger increases in skeletal muscle mass and subsequent expansion of glycogen storage capacity caused by the resistance exercise component [32]. However, in such reports, normalising the volume and workload of essentially different exercise types can prove difficult. Attempts at normalising were made in this 4 month study by estimating the energy expenditure associated with each exercise component [32], while in other studies no normalisation is performed and the volume of combined training is larger than either training regimen alone [23]. Thus, although there are indications of superior glycaemic improvements with combined training, larger exercise volumes may account for some of these changes. Regardless, training of any type improves glycaemic control and should be encouraged, even if the specific training type is deferred to personal choice.

- Both aerobic and resistance exercise acutely enhance insulin sensitivity ( 20\%). Either training regimen further increases insulin sensitivity $(>40 \%)$ and improves $\mathrm{HbA}_{1 \mathrm{c}}$ levels by $\sim 0.4-0.5 \%(\sim 4.1-5.5$ $\mathrm{mmol} / \mathrm{mol})$.

- Combined aerobic and resistance training regimens improve insulin sensitivity $(\sim 70 \%)$ and lower $\mathrm{HbA}_{1 \mathrm{c}}$ levels ( $\sim 0.9 \% ; \sim 10.6 \mathrm{mmol} / \mathrm{mol})$ and these changes are greater than those observed with either training regimen alone.

\section{Effects of training volume and intensity on metabolic adaptations}

Intensity and volume are important factors to consider when initiating a training regimen specifically pertaining to aerobic exercise. An RCT examined the effects of 6 months of aerobic training at different intensities (moderate or vigorous, $40-55 \%$ or $65-85 \%$ peak oxygen consumption $\left[\dot{V} \mathrm{O}_{2 \text { peak }}\right]$, respectively) and volumes (low volume $\sim 5000 \mathrm{~kJ} /$ week or high volume $\sim 8350 \mathrm{~kJ} /$ week) on metabolic variables in overweight, sedentary individuals [33]. Insulin sensitivity, measured during an IVGTT $\sim 24 \mathrm{~h}$ after the final training bout, increased with training (25-65\% vs pre-training), regardless of intensity or volume [33]. However, examination of the same study group showed that, 15 days after the last training bout, insulin sensitivity remained higher only in the moderate, low-volume and vigorous, high-volume aerobic training groups ( $\sim 15 \%$ vs pretraining) [34]. These differences may be accounted for by the greater expansion of glycogen stores and mitochondrial capacity of skeletal muscle in the vigorous, high-volume group, and by longer and more frequent exercise sessions in the moderate, low-volume and vigorous, high-volume groups [34]. In individuals with impaired glucose tolerance, the acute insulin-sensitising effect is stronger after a vigorous bout of exercise than after an isoenergetic, moderate bout of exercise (exercise at $\sim 80 \%$ and $\sim 50 \% \dot{V} \mathrm{O}_{2 \max }$ improved insulin sensitivity by $80 \%$ and $50 \%$, respectively) [35]. However, a parallel trial of 6 months of vigorous and moderate-intensity $(75 \%$ and $50 \% \dot{V} \mathrm{O}_{2 \text { peak }}$, respectively) aerobic training of low- or high-volume (42 and $67 \mathrm{~kJ} \mathrm{~kg}^{-1}$ week $^{-1}$, respectively) exercise reported similar improvements in indices of insulin sensitivity in individuals with impaired fasting glucose for all training regimens [36]. Thus, while higher exercise intensity may confer greater acute insulin sensitisation in population with elevated fasting glucose, effects of longer training may be similar, irrespective of exercise volume and intensity. Several meta-analyses of controlled clinical trials have shown a significant association between improvements in $\mathrm{HbA}_{1 \mathrm{c}}$ levels and exercise intensity, and a modest association with training volume in individuals with type 2 diabetes [18, 37]. While further direct examinations of this relationship are needed, higher exercise intensity and volume may be more

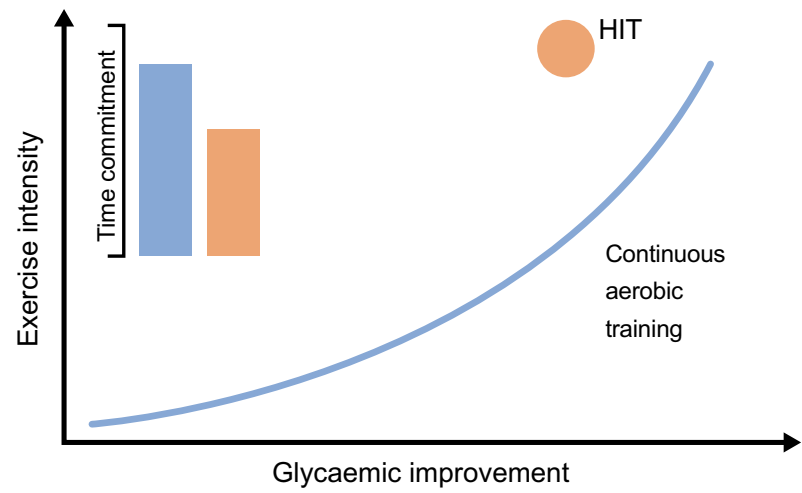

Fig. 3 Glycaemic effects of different training intensity. Beneficial effects of continuous aerobic exercise (blue line) on glycaemic control with exercise intensity. HIT (orange circle) confers superior glycaemic improvement as compared with continuous moderate-intensity training, with a lower time commitment ( $1.5 \mathrm{vs} 2.5 \mathrm{~h} /$ week; inset bars). This figure is available as part of a downloadable slideset 
beneficial for glycaemic control in the type 2 diabetes population (Fig. 3).

Exercise intensity can be modulated by performing an aerobic bout continuously or by alternating high-exertion and low-exertion intervals, known as high-intensity interval training (HIT). Parallel trials have shown that 6 weeks of HIT induces similar athletic performance adaptations to continuous moderate-intensity training in healthy, untrained individuals [38]. Interestingly, HIT improves performance with a lower time commitment compared with continuous moderate-intensity training (1.5 vs $4.5 \mathrm{~h} /$ week, respectively), making HIT an alluring alternative for the engaged contemporary lifestyle [38]. In addition, an acute HIT bout effectively lowers blood glucose levels to a similar extent as an isocaloric bout of moderate intensity [39]. An RCT has shown that 3 months of HIT lowers fasting glucose (by $0.7 \mathrm{mmol} / \mathrm{l}$ ), reduces HOMA-IR (by 25\%) and decreases glycaemic variability (by 5\%) in individuals with type 2 diabetes [40]. These adaptations are comparable to those seen in response to continuous moderate-intensity aerobic training, despite the $40 \%$ lower time commitment ( $\sim 1.5$ vs $2.5 \mathrm{~h} /$ week) and exercise volume for HIT [40]. However, the extent to which untrained individuals can tolerate high-intensity training and their willingness to engage in such regimens are commonly raised concerns. Reports examining exercise tolerance show that untrained individuals do indeed find HIT more exhausting, but also more enjoyable, than continuous moderate-intensity exercise [41]. Thus, owing to its efficacy in improving glycaemic control with lower time commitment and training volume, HIT may be a preferable alternative to more traditional continuous aerobic exercise (Fig. 3). Further studies examining the feasibility of longer training interventions are needed to address the training tolerance and participation rate concerns for individuals with type 2 diabetes.

- Exercise-induced improvements in insulin sensitivity correlate with work intensity. Moderate $(50 \%$ $\left.\dot{V} \mathrm{O}_{2 \max }\right)$ and intense $\left(80 \% \dot{V} \mathrm{O}_{2 \max }\right)$ efforts enhance insulin sensitivity by $50 \%$ and $80 \%$, respectively.

- Moderate-intensity and vigorous exercise training programmes improve glycaemic control.

- Training-induced reductions in $\mathrm{HbA}_{1 \mathrm{c}}$ are coupled to exercise intensity and volume.

\section{Effects of substrate availability on metabolic adaptations to training}

Fuel preference (lipid or carbohydrate) during exercise partly depends on substrate availability and confers different training

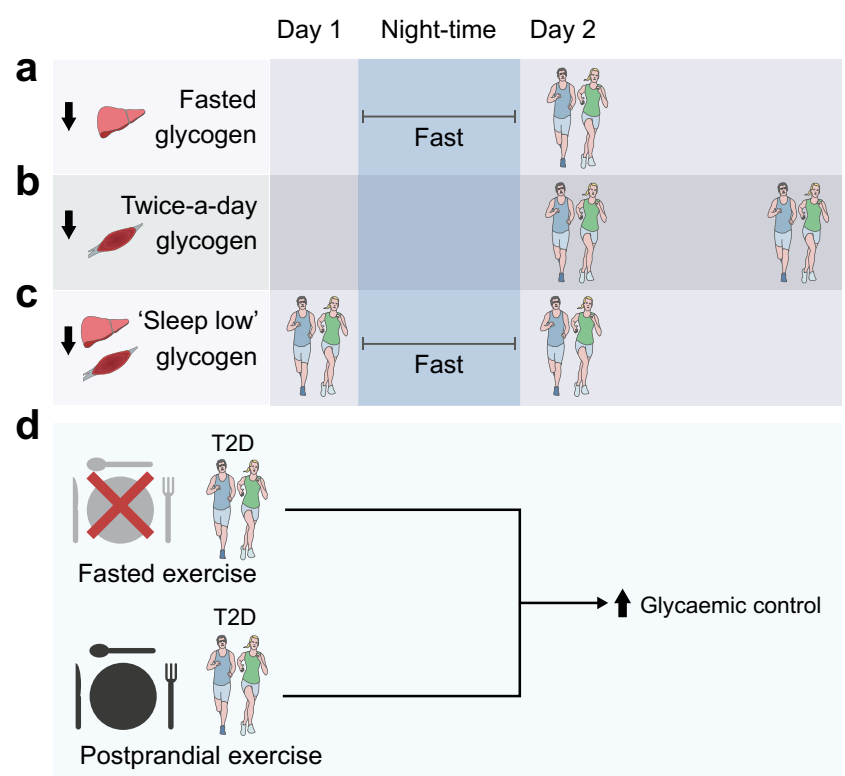

Fig. 4 Training with low carbohydrate availability. Athletes achieve training in a low-carbohydrate state by several methods. (a) Fasting overnight, before an exercise bout, depletes liver glycogen content and lowers carbohydrate availability. (b) Training twice a day depletes skeletal muscle glycogen content during the first exercise bout, allowing the second bout to be initiated with low carbohydrate availability. (c) The 'sleep low' strategy encompasses both, by depleting skeletal muscle glycogen through an evening bout of exercise, and liver glycogen by fasting, before the second bout of exercise in the morning. (d) In individuals with type 2 diabetes, superior glycaemic improvements can be achieved through high-intensity exercise in postprandially or low-intensity exercise under fasted conditions. T2D, type 2 diabetes. This figure is available as part of a downloadable slideset

adaptations to exercise. Athletes put these differences to good use, modulating food intake and training schedules to achieve superior performance. High carbohydrate availability confers the best performance during an activity bout and consumption of carbohydrate-rich beverages reduces symptoms of overtraining [42]. Low carbohydrate availability ('training low') forces the utilisation of lipids as a fuel source and promotes mitochondrial biogenesis and superior adaptations in skeletal muscle oxidative capacity [43, 44]. Traditional approaches of 'training low' are exercising after an overnight fast, with depleted liver glycogen, or exercising twice a day, depleting glycogen stores with the first training bout and initiating the second bout with low skeletal muscle glycogen content (Fig. 4). A novel approach encompasses both strategies and includes performing a HIT bout in the evening followed by an overnight fast, which depletes both skeletal muscle and liver glycogen stores before performing a second exercise bout the following morning (Fig. 4) [43]. This training modality confers superior increases in fatty acid oxidation, fatty acid transporter content and $P G C-1 \alpha$ mRNA expression, as well as improving endurance performance over training with high carbohydrate availability [43, 44]. Therefore, 'training low' is often used to achieve the most beneficial training 
adaptations, while competitive events are performed with high carbohydrate availability to confer the best performance.

Direct examinations of such training strategies in individuals with type 2 diabetes are lacking. However, information on the acute glycaemic response to an exercise bout performed in different nutritional states is available from several crossover RCTs. Insulin sensitivity during an OGTT in healthy individuals is higher immediately after a moderate-intensity cycling bout in a postprandial state than in a fasted state (75\% and 15\% improvement, respectively) [45]. Similarly, a single bout of moderate treadmill walking suppresses high glucose excursions and reduces glycaemic variability in individuals with type 2 diabetes more effectively when performed in a postprandial state than in a fasted state [46]. Converse results were reported in a parallel trial examining two different training modalities (moderate-intensity continuous training and HIT) under different nutritional conditions (fasted and postprandial) in individuals with type 2 diabetes [47]. Glycaemic responses to meals were lower after both exercise types in the fasted state rather than postprandially ( $90 \%$ and $36 \%$ decrease in incremental AUC, respectively), while the most favourable effects on mean glucose concentration $(1.5 \mathrm{mmol} / \mathrm{l}$ decrease), time spent in hyperglycaemia (58\% lower), and fasted glucose levels $(1 \mathrm{mmol} / \mathrm{l}$ decrease $)$ were achieved by HIT under fasted conditions [47]. Hence, the glycaemic response to exercise in different nutritional states may be dependent on the modality and intensity, with a stronger impact of moderate exercise achieved in the postprandial state and superior effects of vigorous exercise attained under fasted conditions (Fig. 4). While these studies provide information regarding the acute responses to exercise in study protocols with divergent carbohydrate availability, they do not fully follow the principles of 'training low' paradigms. Studies examining longer training periods are needed to discern whether the adaptations achieved in the type 2 diabetes population reflect those achieved in healthy individuals, and whether these adaptations are superior to those achieved using conventional training regimens.

- Training with low carbohydrate availability ('training low') is practised by athletes to achieve superior performance improvements.

- The effect of low-intensity exercise on glycaemic control is further enhanced when work bouts are performed in the postprandial state, with high substrate availability.

- The effect of HIT on glycaemic control is further enhanced when work bouts are performed in the fasted state, with low substrate availability.

- The efficacy and safety of longer 'training low' regimens for people with type 2 diabetes warrants further study.

\section{Conclusion}

Selection of the most efficacious training regimen for the management of type 2 diabetes is a complex issue and exercise type, intensity and volume and nutritional status should be considered. Current research indicates that combined resistance and aerobic training regimens and HIT convey superior improvements in glycaemic control [23, 40]. Concerns are often raised about acute side-effects of HIT in the diabetes population [48]. HIT has been applied in cardiovascular disease rehabilitation without increased risk of adverse events [48]. However, the cardiovascular health of the individual needs to be considered when initiating such training regimens. Specific modalities, such as 'training low', are commonly practised by athletes to induce superior physiological adaptations compared with conventional training [43, 44]. However, the efficacy, feasibility and overall effects of such training regimens in individuals with type 2 diabetes are understudied. Notably, the risk of hypoglycaemia could be higher with 'training low', especially in individuals with uncontrolled or insulin-treated type 2 diabetes. Although many exercise regimens are safe for most people, untrained individuals are advised to consult a physician to evaluate whether there are any underlying health issues that may negatively impact full participation. While substantial evidence supports the notion that exercise training improves glycaemic control, further studies are needed to examine the relative advantage of distinct training types and intensities, nutritional status and the interaction of these factors, to improve health outcomes in people with type 2 diabetes. Such investigations would provide additional insight into the application of exercise training regimens to confer the most advantageous metabolic adaptations to combat the rising tide of metabolic disease.

Funding Information Open access funding provided by Karolinska Institutet. Work in the author's laboratories (JZ) is supported by grants from Novo Nordisk Foundation (NNF14OC0011493, NNF14OC0009941, NNF17OC0030088), Swedish Diabetes Foundation (DIA2018-357), Swedish Research Council (2015-00165), the Strategic Research Programme in Diabetes at Karolinska Institutet (2009-1068), the Stockholm County Council (SLL20170159) and the Swedish Research Council for Sport Science (P2019-0140). Additional support was received from the Novo Nordisk Foundation Center for Basic Metabolic Research at the University of Copenhagen (NNF18CC0034900).

Authors' relationships and activities The authors declare that there are no relationships or activities that might bias, or be perceived to bias, their work.

Contribution statement Both authors were responsible for drafting and critically revising the manuscript for important intellectual content. Both authors approved the final version of the manuscript.

Open Access This article is licensed under a Creative Commons Attribution 4.0 International License, which permits use, sharing, adaptation, distribution and reproduction in any medium or format, as long as you give appropriate credit to the original author(s) and the source, provide a link to the Creative Commons licence, and indicate if changes were made. The images or other third party material in this article are 
included in the article's Creative Commons licence, unless indicated otherwise in a credit line to the material. If material is not included in the article's Creative Commons licence and your intended use is not permitted by statutory regulation or exceeds the permitted use, you will need to obtain permission directly from the copyright holder. To view a copy of this licence, visit http://creativecommons.org/licenses/by/4.0/.

\section{References}

1. Hawley JA, Hargreaves M, Joyner MJ, Zierath JR (2014) Integrative biology of exercise. Cell 159(4):738-749. https://doi. org/10.1016/j.cell.2014.10.029

2. Egan B, Zierath JR (2013) Exercise metabolism and the molecular regulation of skeletal muscle adaptation. Cell Metab 17(2):162184. https://doi.org/10.1016/j.cmet.2012.12.012

3. Wahren J, Felig P, Ahlborg G, Jorfeldt L (1971) Glucose metabolism during leg exercise in man. J Clin Invest 50(12):2715-2725. https://doi.org/10.1172/JCI106772

4. Colberg SR, Hagberg JM, McCole SD, Zmuda JM, Thompson PD, Kelley DE (1996) Utilization of glycogen but not plasma glucose is reduced in individuals with NIDDM during mild-intensity exercise. J Appl Physiol (1985) 81(5):2027-2033. https://doi.org/10.1152/ jappl.1996.81.5.2027

5. Bergstrom J, Hultman E (1967) A study of the glycogen metabolism during exercise in man. Scand J Clin Lab Invest 19(3):218228. https://doi.org/10.3109/00365516709090629

6. Gregory S, Wood R, Matthews T, Vanlangen D, Sawyer J, Headley S (2011) Substrate utilization is influenced by acute dietary carbohydrate intake in active, healthy females. J Sports Sci Med 10(1): 59-65

7. Havel RJ, Naimark A, Borchgrevink CF (1963) Turnover rate and oxidation of free fatty acids of blood plasma in man during exercise: studies during continuous infusion of palmitate-1-C $\mathrm{C}^{14}$. J Clin Invest 42(7):1054-1063. https://doi.org/10.1172/JCI104791

8. Costill DL, Daniels J, Evans W, Fink W, Krahenbuhl G, Saltin B (1976) Skeletal muscle enzymes and fiber composition in male and female track athletes. J Appl Physiol 40(2):149-154. https://doi. org/10.1152/jappl.1976.40.2.149

9. Hickson RC (1980) Interference of strength development by simultaneously training for strength and endurance. Eur J Appl Physiol Occup Physiol 45(2-3):255-263. https://doi.org/10.1007/ BF00421333

10. Bouchard C, An P, Rice T et al (1999) Familial aggregation of $\left.\mathrm{VO}_{2 \max }\right)$ response to exercise training: results from the heritage family study. J Appl Physiol (1985) 87(3):1003-1008. https://doi. org/10.1152/jappl.1999.87.3.1003

11. Howald H, Hoppeler H, Claassen H, Mathieu O, Straub R (1985) Influences of endurance training on the ultrastructural composition of the different muscle fiber types in humans. Pflugers Arch 403(4): 369-376. https://doi.org/10.1007/BF00589248

12. Wei M, Gibbons LW, Kampert JB, Nichaman MZ, Blair SN (2000) Low cardiorespiratory fitness and physical inactivity as predictors of mortality in men with type 2 diabetes. Ann Intern Med 132(8): 605-611. https://doi.org/10.7326/0003-4819-132-8-20000418000002

13. Wallberg-Henriksson H, Holloszy JO (1984) Contractile activity increases glucose uptake by muscle in severely diabetic rats. J Appl Physiol Respir Environ Exerc Physiol 57(4):1045-1049. https://doi.org/10.1152/jappl.1984.57.4.1045

14. Kjobsted R, Pedersen AJ, Hingst JR et al (2016) Intact regulation of the AMPK signaling network in response to exercise and insulin in skeletal muscle of male patients with type 2 diabetes: illumination of AMPK activation in recovery from exercise. Diabetes 65(5): 1219-1230. https://doi.org/10.2337/db15-1034

15. Meex RC, Schrauwen-Hinderling VB, Moonen-Kornips E et al (2010) Restoration of muscle mitochondrial function and metabolic flexibility in type 2 diabetes by exercise training is paralleled by increased myocellular fat storage and improved insulin sensitivity. Diabetes 59(3):572-579. https://doi.org/10.2337/db09-1322

16. Vind BF, Pehmoller C, Treebak JT et al (2011) Impaired insulininduced site-specific phosphorylation of TBC1 domain family, member 4 (TBC1D4) in skeletal muscle of type 2 diabetes patients is restored by endurance exercise-training. Diabetologia 54(1):157167. https://doi.org/10.1007/s00125-010-1924-4

17. Zierath JR, Wallberg-Henriksson H (1992) Exercise training in obese diabetic patients. Special considerations. Sports Med 14(3): 171-189. https://doi.org/10.2165/00007256-199214030-00004

18. Boule NG, Kenny GP, Haddad E, Wells GA, Sigal RJ (2003) Metaanalysis of the effect of structured exercise training on cardiorespiratory fitness in type 2 diabetes mellitus. Diabetologia 46(8):10711081. https://doi.org/10.1007/s00125-003-1160-2

19. Boule NG, Haddad E, Kenny GP, Wells GA, Sigal RJ (2001) Effects of exercise on glycemic control and body mass in type 2 diabetes mellitus: a meta-analysis of controlled clinical trials. JAMA 286(10):1218-1227. https://doi.org/10.1001/jama.286.10. 1218

20. Boudou P, De Kerviler E, Vexiau P, Fiet J, Cathelineau G, Gautier J (2000) Effects of a single bout of exercise and exercise training on steroid levels in middle-aged type 2 diabetic men: relationship to abdominal adipose tissue distribution and metabolic status. Diabetes Metab 26(6):450-457

21. Mourier A, Gautier JF, De Kerviler E et al (1997) Mobilization of visceral adipose tissue related to the improvement in insulin sensitivity in response to physical training in NIDDM. Effects of branched-chain amino acid supplements. Diabetes Care 20(3): 385-391. https://doi.org/10.2337/diacare.20.3.385

22. Ronnemaa T, Mattila K, Lehtonen A, Kallio V (1986) A controlled randomized study on the effect of long-term physical exercise on the metabolic control in type 2 diabetic patients. Acta Med Scand 220(3):219-224. https://doi.org/10.1111/j.0954-6820.1986. tb02754.x

23. Sigal RJ, Kenny GP, Boule NG et al (2007) Effects of aerobic training, resistance training, or both on glycemic control in type 2 diabetes - a randomized trial. Ann Intern Med 147(6):357-369. https://doi.org/10.7326/0003-4819-147-6-200709180-00005

24. Christ-Roberts CY, Pratipanawatr T, Pratipanawatr W, Berria R, Belfort R, Mandarino LJ (2003) Increased insulin receptor signaling and glycogen synthase activity contribute to the synergistic effect of exercise on insulin action. J Appl Physiol (1985) 95(6): 2519-2529. https://doi.org/10.1152/japplphysiol.00605.2003

25. Kirwan JP, Solomon TP, Wojta DM, Staten MA, Holloszy JO (2009) Effects of 7 days of exercise training on insulin sensitivity and responsiveness in type 2 diabetes mellitus. Am J Physiol Endocrinol Metab 297(1):E151-E156. https://doi.org/10.1152/ ajpendo.00210.2009

26. Boule NG, Weisnagel SJ, Lakka TA et al (2005) Effects of exercise training on glucose homeostasis: the heritage family study. Diabetes Care 28(1):108-114. https://doi.org/10.2337/diacare.28. 1.108

27. Black LE, Swan PD, Alvar BA (2010) Effects of intensity and volume on insulin sensitivity during acute bouts of resistance training. J Strength Cond Res 24(4):1109-1116. https://doi.org/10. 1519/JSC.0b013e3181cbab6d

28. Dunstan DW, Daly RM, Owen N et al (2002) High-intensity resistance training improves glycemic control in older patients with type 2 diabetes. Diabetes Care 25(10):1729-1736. https://doi.org/10. 2337/diacare.25.10.1729 
29. Holten MK, Zacho M, Gaster M, Juel C, Wojtaszewski JF, Dela F (2004) Strength training increases insulin-mediated glucose uptake, GLUT4 content, and insulin signaling in skeletal muscle in patients with type 2 diabetes. Diabetes 53(2):294-305. https://doi.org/10. 2337/diabetes.53.2.294

30. Ishii T, Yamakita T, Sato T, Tanaka S, Fujii S (1998) Resistance training improves insulin sensitivity in NIDDM subjects without altering maximal oxygen uptake. Diabetes Care 21(8):1353-1355. https://doi.org/10.2337/diacare.21.8.1353

31. Bacchi E, Negri C, Zanolin ME et al (2012) Metabolic effects of aerobic training and resistance training in type 2 diabetic subjects: a randomized controlled trial (the RAED2 study). Diabetes Care 35(4):676-682. https://doi.org/10.2337/dc11-1655

32. Cuff DJ, Meneilly GS, Martin A, Ignaszewski A, Tildesley HD, Frohlich JJ (2003) Effective exercise modality to reduce insulin resistance in women with type 2 diabetes. Diabetes Care 26(11): 2977-2982. https://doi.org/10.2337/diacare.26.11.2977

33. Houmard JA, Tanner CJ, Slentz CA, Duscha BD, McCartney JS, Kraus WE (2004) Effect of the volume and intensity of exercise training on insulin sensitivity. J Appl Physiol (1985) 96(1):101106. https://doi.org/10.1152/japplphysiol.00707.2003

34. Bajpeyi S, Tanner CJ, Slentz CA et al (2009) Effect of exercise intensity and volume on persistence of insulin sensitivity during training cessation. J Appl Physiol (1985) 106(4):1079-1085. https://doi.org/10.1152/japplphysiol.91262.2008

35. Rynders CA, Weltman JY, Jiang B et al (2014) Effects of exercise intensity on postprandial improvement in glucose disposal and insulin sensitivity in prediabetic adults. J Clin Endocrinol Metab 99(1):220-228. https://doi.org/10.1210/jc.2013-2687

36. Slentz CA, Bateman LA, Willis LH et al (2016) Effects of exercise training alone vs a combined exercise and nutritional lifestyle intervention on glucose homeostasis in prediabetic individuals: a randomised controlled trial. Diabetologia 59(10):2088-2098. https://doi.org/10.1007/s00125-016-4051-z

37. Snowling NJ, Hopkins WG (2006) Effects of different modes of exercise training on glucose control and risk factors for complications in type 2 diabetic patients: a meta-analysis. Diabetes Care 29(11):2518-2527. https://doi.org/10.2337/dc06-1317

38. Burgomaster KA, Howarth KR, Phillips SM et al (2008) Similar metabolic adaptations during exercise after low volume sprint interval and traditional endurance training in humans. J Physiol 586(1): 151-160. https://doi.org/10.1113/jphysiol.2007.142109

39. Shambrook P, Kingsley MI, Wundersitz DW, Xanthos PD, Wyckelsma VL, Gordon BA (2018) Glucose response to exercise in the post-prandial period is independent of exercise intensity.
Scand J Med Sci Sports 28(3):939-946. https://doi.org/10.1111/ sms.12999

40. Winding KM, Munch GW, Iepsen UW, Van Hall G, Pedersen BK, Mortensen SP (2018) The effect on glycaemic control of lowvolume high-intensity interval training versus endurance training in individuals with type 2 diabetes. Diabetes Obes Metab 20(5): 1131-1139. https://doi.org/10.1111/dom.13198

41. Bartlett JD, Close GL, MacLaren DP, Gregson W, Drust B, Morton JP (2011) High-intensity interval running is perceived to be more enjoyable than moderate-intensity continuous exercise: implications for exercise adherence. J Sports Sci 29(6):547-553. https:// doi.org/10.1080/02640414.2010.545427

42. Halson SL, Lancaster GI, Achten J, Gleeson M, Jeukendrup AE (2004) Effects of carbohydrate supplementation on performance and carbohydrate oxidation after intensified cycling training. $\mathrm{J}$ Appl Physiol (1985) 97(4):1245-1253. https://doi.org/10.1152/ japplphysiol.01368.2003

43. Lane SC, Camera DM, Lassiter DG et al (2015) Effects of sleeping with reduced carbohydrate availability on acute training responses. J Appl Physiol (1985) 119(6):643-655. https://doi.org/10.1152/ japplphysiol.00857.2014

44. Marquet LA, Brisswalter J, Louis J et al (2016) Enhanced endurance performance by periodization of carbohydrate intake: "Sleep low" strategy. Med Sci Sports Exerc 48(4):663-672. https://doi. org/10.1249/MSS.0000000000000823

45. Edinburgh RM, Hengist A, Smith HA et al (2018) Preexercise breakfast ingestion versus extended overnight fasting increases postprandial glucose flux after exercise in healthy men. Am J Physiol Endocrinol Metab 315(5):E1062-E1074. https://doi.org/ 10.1152/ajpendo.00163.2018

46. Nygaard H, Ronnestad BR, Hammarstrom D, Holmboe-Ottesen G, Hostmark AT (2017) Effects of exercise in the fasted and postprandial state on interstitial glucose in hyperglycemic individuals. J Sports Sci Med 16(2):254-263

47. Terada T, Wilson BJ, Myette-Cote E et al (2016) Targeting specific interstitial glycemic parameters with high-intensity interval exercise and fasted-state exercise in type 2 diabetes. Metabolism 65(5):599 608. https://doi.org/10.1016/j.metabol.2016.01.003

48. Rognmo O, Moholdt T, Bakken $\mathrm{H}$ et al (2012) Cardiovascular risk of high- versus moderate-intensity aerobic exercise in coronary heart disease patients. Circulation 126(12):1436-1440. https://doi. org/10.1161/CIRCULATIONAHA.112.123117

Publisher's note Springer Nature remains neutral with regard to jurisdictional claims in published maps and institutional affiliations. 